\title{
Barreiras e facilitadores percebidos por pessoas com deficiência praticantes de esportes
}

\author{
Barriers and facilitators perceived by people with disabilities that practice sports
}

\section{Barreras y facilitadores percibidos por personas con discapacidad que practican deportes}

\author{
Grazieli Maria Biduski ${ }^{\mathrm{I}}$, Morgana Lunardi ${ }^{\mathrm{II}}$, Mateus Rossato ${ }^{\mathrm{III}}$, Josefina Bertoli ${ }^{\mathrm{IV}}$, Cintia de la Rocha \\ Freitas $^{\mathrm{V}}$, Bruna Barboza Seron ${ }^{\mathrm{VI}}$
}

\begin{abstract}
Resumo
Considerando a importância da inserção de pessoas com deficiência no esporte, objetivamos identificar as barreiras e os facilitadores para a prática esportiva de pessoas com deficiência inseridas nos projetos de extensão da Universidade Federal de Santa Catarina (Brasil). Para determinar as barreiras e os facilitadores foi aplicado um questionário a 19 participantes. Usou-se o teste qui-quadrado para avaliar a associação entre barreiras e facilitadores $(\mathrm{p}<0,05)$. Os indicadores estatisticamente associados como facilitadores para a prática esportiva de pessoas com deficiência incluem a simpatia e conhecimento profissional, apoio da família e amigos e a ausência de constrangimento à prática. A presença de lesões, o clima e a distância do local da prática foram associados como barreiras. Desse modo, propiciar um ambiente de elevado apoio social, estabelecer cuidados para evitar lesões físicas e oferecer programas esportivos descentralizados, na cidade, parecem ser medidas importantes para a efetiva participação de pessoas com deficiência no esporte.
\end{abstract}

Palavras-chave: Pessoas com deficiência; Acessibilidade arquitetônica; Instalações Esportivas e Recreacionais

\begin{abstract}
Considering the importance of including people with disabilities in sport, we aim to identify barriers and facilitators for the practice of sports by people with disabilities and that are included in the extension projects of the Federal University of Santa Catarina (Brazil). To determine barriers and facilitators, a questionnaire was applied to nineteen participants Chi-square test was used to assess the association between barriers and facilitators $(\mathrm{p}<0,05)$. Indicators statistically associated as facilitators for the practice of sports by people with disabilities include sympathy and professional knowledge, support from family and friends, and the absence of embarrassment to the practice. The presence of injuries, climate and distance from the practice site were associated as barriers. Thus, providing an environment of high social support, establishing care to avoid physical injuries and offering decentralized sports programs, in the city, seem to be important measures for effective people participation with disabilities in sports.
\end{abstract}

Keywords: Disabled Persons; Architectural Accessibility; Sports and Recreational Facilities

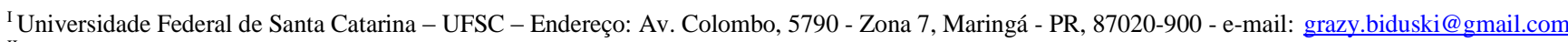

II Universidade Federal de Santa Catarina - UFSC - e-mail: morganalunardi.edf@gmail.com

III Universidade Federal do Amazonas - UFAM - e-mail: rossato.mateus@ gmail.com

${ }^{\text {IV }}$ Universidade Estadual Paulista - Unesp - e-mail: josefinabv85@gmail.com

v Universidade Federal de Santa Catarina - UFSC - e-mail: cintiadelarocha@gmail.com

${ }^{\mathrm{VI}}$ Universidade Federal de Santa Catarina - UFSC - e-mail: bruna89@msn.com
} 


\section{Resumen}

Considerando la importancia de la inserción de personas con discapacidad en el deporte, el objetivo de este estudio fue identificar las barreras y facilitadores para la práctica deportiva de personas con discapacidad insertadas en los proyectos de extensión de la Universidad Federal de Santa Catarina (Brasil). Para barreras y facilitadores se aplicó un cuestionario a 19 participantes. Se utilizó el test Qui-cuadrado para evaluar la asociación entre barreras y facilitadores $(\mathrm{p}<0,05)$. Los indicadores estadísticamente asociados como facilitadores para la práctica de deportes de personas con discapacidad incluyen la simpatía y conocimientos profesional, apoyo de la familia y amigos y la ausencia de vergüenza para la práctica. Presencia de lesiones, el clima y la distancia del local de la práctica fueron asociados como barreras. Siendo así, proporcionar un ambiente de gran apoyo social, establecer cuidados para prevenir lesiones físicas y ofrecer programas deportivos descentralizados en la ciudad, parecen ser medidas importantes para una efectiva participación de personas con discapacidad en el deporte.

Palabras clave: Personas con Discapacidad; Accesibilidad Arquitectónica; Instalaciones Deportivas y Recreativas

\section{Introdução}

Dados da World Health Organization (WHO) apontam mais de um bilhão de pessoas com algum tipo de deficiência ao redor do mundo (WHO, 2016). Segundo o Instituto Brasileiro de Estatística e Geografia (IBGE, 2010), a cidade de Florianópolis tem uma população estimada em 500 mil pessoas e destas, 25.785 apresentam alguma deficiência. Pessoas com deficiência apresentam baixos indicativos de saúde, de escolaridade e, consequentemente, poucas oportunidades de participação social (WHO, 2016). A inclusão das pessoas com deficiência em programas de exercícios físicos ou na prática esportiva tem sido recomendada para melhorar os índices de saúde (MARTIN, 2013). Amplamente conhecida, a prática regular de exercícios físicos está associada à melhora da qualidade de vida e à maior interação social, além de ser uma ótima alternativa para prevenção e tratamento de doenças, independentemente da condição física do indivíduo (CARDOSO, 2011).

A prática de exercícios físicos e/ ou esportivos por pessoas com deficiência é de extrema importância, tendo em vista que essa população tem uma maior predisposição ao sedentarismo (ANDERSON; HEYNE, 2010). Uma vez inseridas em um novo contexto, principalmente em esportes coletivos, essas pessoas tendem a melhorar sua auto percepção, o que, de fato, eleva a autoestima, que, por sua vez, contribui positivamente nos aspectos psicológicos (MARTIN, 2013). Contudo, apesar dos benefícios que a prática esportiva promove, ainda são observados uma baixa inserção e um grande índice de desistências por parte dessa população (VAN der PLOEG et al., 2007; MARMELEIRA et al., 2018).

A dificuldade em se inserir ou manter-se em um programa de exercícios ou prática esportiva pode estar relacionada às diversas barreiras enfrentadas no dia a dia. As barreiras podem estar atreladas às atitudes negativas por parte da sociedade o que pode causar constrangimento durante a prática (ROLFE et al., 2009); às próprias condições físicas; a um número reduzido de programas de exercícios 
específicos; ao desânimo ou cansaço (falta de energia); à falta de interesse; à inabilidade física; a não obtenção de recurso financeiro (SERON et al.; 2015); ou mesmo à falta de locais e espaços adequados disponíveis para a prática de exercícios físicos ou esportivos (RIMMER et al., 2004).

Por isso, pensando em reduzir essas barreiras, um dos objetivos específicos da Política Nacional de Promoção da Saúde é "contribuir para a adoção de práticas sociais e de saúde centradas na equidade, na participação e no controle social, visando reduzir as desigualdades sistemáticas, injustas e evitáveis, com respeito às diferenças [...] relacionadas às pessoas com deficiências e necessidades especiais" (BRASIL, 2010). Reflexões sobre as dificuldades que essa população enfrenta ao acesso provocado pelas barreiras devem ser discutidas, pois contribuem para o repensar das práticas e de ações em ambientes públicos que possam favorecer a promoção de saúde e qualidade de vida dessas pessoas (LAMÔNICA et al., 2008).

Embora o entendimento das barreiras enfrentadas seja importante, a compreensão de quais facilitadores, ou seja, o que contribui para que as pessoas permaneçam engajadas na prática esportiva, pode trazer informações que direcionem as instituições envolvidas com estes grupos de pessoas para a adesão e permanência nos programas esportivos. Nesse sentido, estudos anteriores reportaram que alguns facilitadores percebidos para a prática esportiva estavam relacionados com um espaço que forneça equipamentos adequados à prática (RIMMER et al., 2004), presença de profissionais capacitados (SCELZA et al., 2005) e apoio de amigos e familiares (SERON et al., 2015). A percepção de melhora da independência e a compreensão dos benefícios que a prática de atividade física promove também foram elencados como facilitadores pelas pessoas com deficiência (WILLIAMS et al., 2014). Diminuir as barreiras e aumentar os facilitadores deve ser o objetivo das instituições e organizações esportivas que desenvolvem projetos para esta população.

Com base nesses conhecimentos, estratégias podem ser realizadas com a finalidade de modificar o atual contexto (JAARSMA et al., 2014). Um exemplo de programas voltados para pessoas com deficiência, no âmbito esportivo, é o projeto de extensão promovido pelo curso de Educação Física do Centro de Desportos na Universidade Federal de Santa Catarina (UFSC). As atividades esportivas são oferecidas há mais de 20 anos para essa população (CENTRO DE DESPORTOS, 2018). Apesar da longa trajetória, o projeto carece de informações sobre fatores que afetam negativamente ou estimulam a continuidade da prática esportiva, a fim de identificar e discutir possíveis mudanças para melhorar o ambiente esportivo. Assim, o objetivo deste estudo foi identificar as barreiras e os facilitadores pessoais e ambientais para a prática esportiva para pessoas com deficiência inseridas nos projetos de extensão da UFSC. 


\section{Material e Métodos}

Este é um estudo descritivo, com abordagem quantitativa e transversal. A coleta de dados foi realizada a partir da autorização dos professores responsáveis pelos projetos de extensão e do consentimento dos participantes através do Termo de Consentimento Livre e Esclarecido, respeitando os princípios éticos que orientam os termos da Resolução 466/2012 do Conselho Nacional de Saúde.

Participaram do estudo 19 pessoas com deficiência, sendo duas mulheres e 17 homens, com média de idade de $41( \pm 10,2)$ anos, participantes dos projetos de extensão da UFSC, moradores da Grande Florianópolis/ SC. A maior parte desses participantes são vinculados à Associação Catarinense de Esportes Adaptados (ACESA). Para fazer parte da pesquisa, os participantes deveriam ter acima de 18 anos, apresentar alguma deficiência e participar dos projetos de extensão para pessoas com deficiência há mais de seis meses. O poder amostral a posteriori foi calculado considerando $\alpha=5 \%, \beta=80 \%$ e $q u i$ quadrado entre os papéis desempenhados e os domínios do estilo de vida, ressentindo-se de um alto tamanho de efeito $(d=0,94)$, por meio do software $G *$ Power versão 3.1.9.2.

Uma anamnese elaborada pelos próprios autores do estudo foi utilizada, com a finalidade de obter informações referentes à idade, gênero, tipo de deficiência, tempo de prática e modalidade esportiva praticada. Para verificar as barreiras e facilitadores, utilizou-se um questionário adaptado de Seron et al. (2015), no qual se propõe caracterizar os fatores pessoais e ambientais observados pelos participantes durante a prática esportiva. O questionário foi composto por 24 perguntas fechadas e subdividido em indicadores pessoais e ambientais.

Foram listados, como pessoais, os seguintes indicadores: simpatia e conhecimento profissional; apoio da família e de amigos; presença de lesões; ausência de constrangimento durante realização da prática esportiva; disposição para a prática; atitude das demais pessoas; presença de doenças; custo financeiro; preocupação com aparência; medo de sofrer lesões; e limitações físicas próprias. Em relação aos indicadores ambientais, foram citados: clima (sol, chuva, vento, etc.); distância do local da prática; equipamentos disponíveis; horário para a prática; divulgação de informações referentes ao esporte; transporte; jornada de trabalho; tarefas domésticas; segurança do ambiente; acessibilidade arquitetônica; e falta de espaços disponíveis.

O preenchimento do questionário foi realizado pelo próprio indivíduo, acompanhado dos pesquisadores responsáveis, para sanar as dúvidas, sempre que necessário. Para os participantes com deficiência visual, a pesquisadora responsável que não tinha nenhum envolvimento com os participantes, 
não havendo, dessa forma, nenhum conflito de interesse, fez a leitura das perguntas e assinalou as respectivas respostas.

Os dados foram apresentados em média, desvio padrão, valores relativos e absolutos. Foi utilizado o teste de qui-quadrado para verificar as possíveis associações entre as características demográficas e os indicadores de barreiras e facilitadores. Ainda foi realizado o teste de comparação entre duas proporções, para verificar possíveis diferenças significativas entre as frequências de respostas dos sujeitos, com base nos indicadores "ajuda ou atrapalha a prática de atividade física". As análises foram feitas por meio do programa Statistical Package for the Social Science (SPSS), versão 17.0.

\section{Resultados}

Participaram do estudo 19 pessoas com deficiência, 2 mulheres e 17 homens, com média de idade

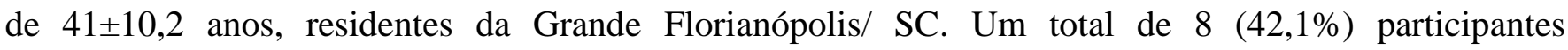
apresentavam deficiência visual, 6 (31,6\%) lesão medular, 2 (10,5\%) amputação, 2 (10,5\%) sequela de

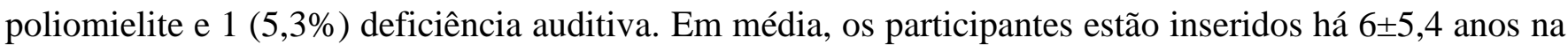
prática esportiva. As modalidades esportivas praticadas pelos participantes eram: natação (n=2; 10,5\%), tênis em cadeira de rodas $(n=2 ; 10,5 \%)$, goalball $(n=8 ; 42,1 \%)$ e handebol em cadeira de rodas $(n=7$; $36,8 \%$ ). Na tabela 1, estão apresentados os indicadores pessoais, e, na tabela 2, os indicadores ambientais associados às barreiras ou facilitadores para a prática esportiva na UFSC.

Tabela 1 - Indicadores pessoais de barreiras ou facilitadores à prática esportiva na UFSC, Florianópolis, Santa Catarina, Brasil, 2017.

\begin{tabular}{lccc}
\hline \multicolumn{1}{c}{ Indicadores pessoais } & Facilitadores & Barreiras & p (Qui-quadrado) \\
\hline Simpatia do profissional & $\mathrm{n}(\%)$ & $\mathrm{n}(\%)$ & \\
Conhecimento profissional & $19(100,0)$ & $00(0,00)$ & $\mathbf{0 , 0 0 0}^{*}$ \\
Apoio da família & $17(94,44)$ & $01(5,56)$ & $\mathbf{0 , 0 0 0}^{*}$ \\
Apoio dos amigos & $16(84,21)$ & $03(15,79)$ & $\mathbf{0 , 0 0 4}^{*}$ \\
Presença de lesões & $17(89,47)$ & $02(10,53)$ & $\mathbf{0 , 0 0 1}^{*}$ \\
Ausência de constrangimento durante a & $02(10,53)$ & $17(89,47)$ & $\mathbf{0 , 0 0 1 *}$ \\
prática & $17(89,47)$ & $02(10,53)$ & $\mathbf{0 , 0 0 1 *}$ \\
Disposição para a prática & $14(73,68)$ & $05(26,32)$ & 0,064 \\
Atitude das demais pessoas & $14(73,68)$ & $05(26,32)$ & 0,064 \\
Presença de doenças & $09(47,37)$ & $10(52,63)$ & 1,000 \\
Falta de recurso financeiro & $07(36,84)$ & $12(63,16)$ & 0,359 \\
\hline
\end{tabular}




\begin{tabular}{|c|c|c|c|}
\hline Preocupação com aparência & $14(73,68)$ & $05(26,32)$ & 0,064 \\
\hline Medo de se lesionar & $08(42,11)$ & $11(57,89)$ & 0,648 \\
\hline Limitações físicas & $09(52,94)$ & $08(47,06)$ & 0,185 \\
\hline
\end{tabular}

* Associação significativa dos indicadores pessoais como barreiras ou facilitadores $(\mathrm{p}<0,05)$.

Fonte: Autores

Tabela 2 - Indicadores ambientais de barreiras ou facilitadores para a prática esportiva na UFSC, Florianópolis, Santa Catarina, Brasil, 2017.

\begin{tabular}{lccc}
\hline \multicolumn{1}{c}{ Indicadores ambientais } & $\begin{array}{c}\text { Facilitadores } \\
\mathrm{n}(\%)\end{array}$ & $\begin{array}{c}\text { Barreiras } \\
\mathrm{n}(\%)\end{array}$ & $\mathrm{p}$ (Qui-quadrado) \\
\hline Clima (vento, frio, calor, etc.) & $03(15,79)$ & $16(84,21)$ & $\mathbf{0 , 0 0 4} *$ \\
Distância do local de prática & $03(15,79)$ & $16(84,21)$ & $\mathbf{0 , 0 0 4 *}$ \\
Equipamentos disponíveis & $14(73,68)$ & $05(26,32)$ & 0,064 \\
Horário para a prática & $12(63,16)$ & $07(36,84)$ & 0,359 \\
Divulgação de informações & $14(73,68)$ & $05(26,32)$ & 0,064 \\
Transporte & $07(36,84)$ & $12(63,16)$ & 0,359 \\
Jornada de trabalho & $12(63,16)$ & $07(36,84)$ & 0,359 \\
Tarefas domésticas & $14(73,68)$ & $05(26,32)$ & 0,064 \\
Segurança do ambiente & $07(36,84)$ & $12(63,16)$ & 0,359 \\
Acessibilidade arquitetônica & $08(42,11)$ & $11(57,89)$ & 0,648 \\
Falta de espaço para prática & $08(42,11)$ & $11(57,89)$ & 0,648 \\
\hline
\end{tabular}

* Associação significativa dos indicadores ambientais como barreiras ou facilitadores $(\mathrm{p}<0,05)$.

Fonte: Autores

\section{Discussão}

O objetivo do presente estudo foi identificar barreiras e facilitadores, tanto pessoais quanto ambientais, percebidos por pessoas com deficiência inseridas nos projetos de extensão da UFSC, que promove a prática esportiva. De acordo com os achados, os principais indicadores significativos associados aos facilitadores para a prática esportiva incluem simpatia do profissional, conhecimento profissional, apoio da família e de amigos e ausência de constrangimento durante a prática $(\mathrm{p}<0,05)$.

Similar resultado foi apontado por McLoughlin et al. (2017), os quais observaram que, mesmo atletas envolvidos com alto rendimento, reconhecem que a influência da família, do Coach, dos demais profissionais envolvidos, dos amigos e companheiros de equipe, exerce total influência na continuidade e no envolvimento com a prática esportiva. Da mesma forma, Becerra et al. (2019), observaram o apoio da família, de amigos e de profissionais da saúde, além do ambiente favorável para a prática esportiva, fatores que mais influenciam positivamente a adesão e a motivação de atletas de rugby em cadeira de rodas.

Para os atletas de rugby em cadeira de rodas inseridos em um projeto de extensão da Universidade Federal da Paraíba, o envolvimento das pessoas com deficiência na prática esportiva promove a 
integração e a socialização pelas trocas de ideias e experiências vividas, e esse convívio fortalece as relações pessoais no dia a dia (AMARAL FILHO et al., 2013). A boa intervenção por parte do profissional de saúde (VAN der PLOEG et al., 2004), aliada à positiva influência social, opinião e comportamento da sociedade, são fatores determinantes para o envolvimento das pessoas com deficiência nos programas de exercícios ou esportivos (MACKENZI et al., 2021). Facilitadores como, gostar das atividades realizadas, sentir prazer na prática, não apresentar timidez ou preguiça, dispor de tempo e não vivenciar experiências negativas foram elencados por 1191 pessoas com deficiência intelectual que frequentavam as Associação de Pais e Amigos dos Excepcionais (APAES) do Rio Grande do Sul (JUNG et al., 2017).

Dessa forma, fica claro que o apoio social de forma geral, principalmente das pessoas mais próximas, contribui para a permanência das pessoas com deficiência nesses espaços. A socialização entre as pessoas com deficiência é benéfica para a saúde física e mental, pois o fato das pessoas se sentirem pertencentes a um determinado grupo faz com que queiram permanecer inseridas nesse contexto. Sentirse pertencente a um grupo e estar inserido em uma modalidade esportiva auxilia a modificar a percepção do indivíduo sobre sua própria deficiência (SILVA et al., 2013). Esse ponto dialoga com os resultados do presente estudo, haja vista que o fato de os participantes não sentirem constrangimento durante a prática esportiva foi um indicador favorável à participação e à continuidade no meio esportivo. Ademais, os participantes do estudo estavam envolvidos nos projetos de extensão da UFSC em média, há seis anos, o que pode, de fato, ter contribuído para esta percepção positiva frente aos benefícios obtidos com o esporte.

Esse considerável tempo de envolvimento na prática esportiva pode ter contribuído para que os participantes do estudo, embora não tenham apresentado valores significativos, tenham percebido muito dos indicadores pessoais e ambientais (equipamentos disponíveis, horários para a prática, jornada de trabalho, tarefas domésticas, disposição para a prática, limitações físicas, preocupação com a aparência e atitudes das demais pessoas) como algo que não interfere na prática esportiva. Esse fato merece certo destaque, já que diversas pesquisas apontam esses mesmos indicadores como barreiras (JAARSMA et al., 2014, SCELZA et al., 2005; BECERRA et al., 2019).

Segundo Bragaru et al. (2013), quando as pessoas estão inseridas em uma prática esportiva, elas concentram-se mais, no que diz respeito aos aspectos físicos e psicológicos, nas vantagens de estarem envolvidas com o esporte e nos benefícios que o mesmo promove do que nas barreiras encontradas para a prática. Para Martin (2013), uma barreira pode ser irrelevante para uns e muito significativa para outros, 
no entanto, a prática esportiva ou o engajamento em programas de exercícios tornam as pessoas mais fortes, e as barreiras, vistas antes da prática, deixam de existir depois da adesão.

Dessa forma, sobre a perspectiva de barreiras percebidas, os participantes do presente estudo reportaram a distância do local de prática, o clima e a presença de lesões como fatores significativos que afetam a prática esportiva. A distância do local da prática reflete a falta de ofertas descentralizadas de programas esportivos que pensem na pessoa com deficiência. Jung et al. (2017) observou que uma das barreiras relatadas pelos participantes de seu estudo tinha relação com a falta de projetos sociais próximos às residências. Muitos dos projetos de prática esportiva voltados para as pessoas com deficiência ocorrem em lugares específicos (universidades ou espaços pouco voltados para essa população) e esse menor número de espaços pode ser realmente uma barreira. No estudo de McLoughlin et al. (2017), mesmo os atletas de nível competitivo, com deficiência reportaram que a distância afeta o envolvimento e a participação de demais pessoas à prática esportiva aumentando essa desigualdade de oportunidade. Esse apontamento tem grande relevância, pois, segundo os mesmos atletas, o engajamento das pessoas no esporte se torna inviável, principalmente aquelas com deficiência que residem em cidades do interior ou não pertencem aos grandes centros urbanos.

Assim, com baixo número de locais de práticas ou de projetos, as pessoas precisariam percorrer, muitas vezes, grandes distâncias, para terem oportunidade de se inserir em uma prática esportiva, uma realidade contrastante quando comparada as pessoas sem deficiência que têm oferta de práticas esportivas em locais adequados e em diversos lugares. Em Florianópolis, além das práticas paradesportivas ofertadas na UFSC, há oportunidades de práticas em outros locais da cidade. As associações como a Associação Catarinense para Integração do Cego (ACIC), a Associação Florianopolitana de Deficientes Físicos (AFLODEF), a Associação Surf Sem Fronteiras (ASSF) e a já mencionada ACESA ofertam práticas variadas. No entanto, práticas específicas são ofertadas em locais específicos, não havendo por exemplo, diversos lugares oferecendo uma mesma modalidade, fato que pode ainda prejudicar o engajamento.

As associações são as principais instituições de vínculo das pessoas com deficiência. Nessa perspectiva, um recente estudo de Monforte et al. (2021) aponta que as barreiras e os facilitadores dependem do contexto e, portanto, não precedem as circunstâncias em que emergem. Assim, ao investigarem diferentes contextos, observaram que universitários com deficiência da Espanha apontam maior acolhimento nas associações do que na Universidade. Essa discussão levantada pelo estudo abre margem para reflexões, pois, se, por um lado, a descentralização de oportunidades de prática é fundamental para aumentar o acesso, por outro, os diferentes cenários de ofertas devem, assim como as associações, suportarem-se com ambientes narrativos nos quais a identidade da deficiência é fortalecida. 
O clima, elencado como barreira, pode estar atrelado também à distância do local para a prática. As condições climáticas podem expor as pessoas a situações desfavoráveis influenciando na decisão do participante de se deslocar até o local para a prática esportiva (SERON et al., 2015). Apesar de toda independência conquistada, algumas situações não percebidas por pessoas sem deficiência, como a chuva, as quais afetam significativamente a qualidade de vida das pessoas com deficiência.

Embora terem sido reportadas poucas barreiras no presente estudo, a presença de lesão afeta de forma significativa a prática esportiva. A participação no esporte, se não orientada, pode aumentar o risco de lesões e, dependendo da lesão, o indivíduo ficará afastado do esporte e das demais tarefas diárias por longos períodos de tempo (LJUNGQVIST et al., 2009). Segundo estudos de Buffart et al. (2009) e Lape et al. (2017), a presença de lesões é uma preocupação recorrente em esportistas com deficiência, haja vista o medo de que lesões os impeçam de ter um futuro no esporte. As informações obtidas referentes às lesões devem ser consideradas pelos profissionais responsáveis pelo acompanhamento desses atletas, seja em nível competitivo ou recreacional. Muitos dos usuários em cadeira de rodas, por exemplo, já possuem lesões devido ao estresse das atividades do dia a dia. Assim, compreender e reduzir os riscos de uma lesão e ter conhecimento da epidemiologia da lesão implica criar estratégias eficazes na prevenção de futuras lesões, contribuindo, dessa forma, no desempenho esportivo e na melhora da qualidade de vida dessas pessoas (FAGHER; LEXELL, 2014).

Apesar de as barreiras não terem sido elencadas em grande número pelos participantes do presente estudo, outras pesquisas identificaram, como barreiras, a dificuldade financeira (SCELZA et al., 2005; BECERRA et al., 2019), a falta de orientação qualificada dos profissionais para a prática (SHIELDS et al., 2012) e, até mesmo, a não compreensão da importância do exercício e do esporte para a saúde (RIMMER et al., 2004). Acrescenta-se a essas barreiras, acessibilidade e transporte (ELLIS et al., 2007; JAARSMA et al., 2014), estruturas inadequadas (ELLIS et al., 2007), a falta de equipamentos disponíveis e adaptados (PALMA et al., 2020), escassez de programas específicos e as próprias limitações físicas (SERON et al., 2015). De certa forma, pode ser observado que as barreiras elencadas em um estudo e não no outro dependem muito do contexto de cada grupo, ou seja, retratam a realidade específica de determinada região. Vale ressaltar, também, que as barreiras e os facilitadores percebidos dependem muito dos objetivos das pessoas e quão motivadas estão para dar continuidade à prática, apesar dos desafios diários.

Além da investigação principal, destaca-se, no presente estudo, a baixa inserção de mulheres, mesmo quando as modalidades esportivas são variadas e gratuitas. De acordo com Marmeleira et al. (2018), as barreiras apontadas pelas mulheres para a não adesão a prática de exercícios e/ ou esportiva, 
estão relacionadas às questões atitudinais das outras pessoas em relação a sua deficiência e à própria aceitação pessoal. As mulheres relatam ter uma autopercepção das suas habilidades e competências físicas inferiores às dos homens (HAYES et al., 1999), além de baixa autoestima, que limita a participação no desporto (BRITTAIN, 2004). Para Marmeleira et al. (2018), as mulheres com deficiência visual parecem ser ainda mais dependentes de outras pessoas no que se refere ao deslocamento, pois não ter quem as leve até o local da prática é uma grande barreira. A menor adesão das mulheres ao ambiente esportivo deve ser considerada e investigada em futuros estudos, a fim de modificar o atual contexto.

De forma geral, os resultados encontrados durante o presente estudo podem estar relacionados ao fato dessa população, em específico, não enfatizar barreiras ambientais ou pessoais, mas, sim, valorizar a possibilidade de realizar uma prática esportiva. É importante ressaltar que os facilitadores encontrados foram relacionados aos indicadores pessoais, e as barreiras, atreladas aos fatores ambientais. Então, o fato de as pessoas com deficiência estarem inseridas em uma prática esportiva há muitos anos e sentirem assistidas pelos profissionais as motivam à continuidade, ao passo que, as barreiras percebidas, relacionadas a indicadores ambientais, não dependem de atitudes pessoais, mas envolvem questões de políticas públicas, fatores externos às suas competências.

O fato de, no presente estudo, terem sido apontados mais facilitadores do que barreiras, por parte dos participantes do projeto de extensão da UFSC, reforça a importância da própria prática para percepções positivas sobre a mesma. Entretanto, essa percepção não deve minimizar a preocupação em se identificar e solucionar possíveis barreiras, a fim de contribuir com a permanência dos indivíduos na prática esportiva e facilitar o ingresso de novos participantes.

\section{Conclusão}

Os resultados do presente estudo indicam que pessoas com deficiência praticantes de esportes na UFSC percebem mais facilitadores do que barreiras para o engajamento na prática. Como destaque, os facilitadores percebidos são relacionados a fatores pessoais, enquanto as barreiras relacionaram-se mais aos fatores ambientais. Assim, propiciar um contexto de elevado apoio social, estabelecer cuidados para evitar lesões físicas e oferecer programas esportivos descentralizados na cidade parecem ser medidas importantes para a efetiva participação de pessoas com deficiência no âmbito esportivo. 


\section{Referência}

AMARAL FILHO, H. J. A.; SOUTO, E. C.; SANTOS, E. A.; DANTAS, I. R. V.; TIBURTINO, M. J. S. Barreiras e facilitadores para a prática de atividade física nos atletas do rugby em cadeira de rodas. Centro de Ciências da Saúde, Departamento de Educação Física, PROBEX. Disponível em: http://www.prac.ufpb.br/enex/trabalhos/6CCSDEFPROBEX2013187.pdf. Acesso em: 15 fev. 2020.

ANDERSON, L. S; HEYNE, L. A. Physical activity for children and adults with disabilities: an issue of "amplified" importance. Disability and Health Journal, v. 3, n. 2, p. 71-73, apr. 2010.

BECERRA, M.; MANZINI, M. G; MARTINEZ, C. M. S. Percepção de atletas do rugby em cadeira de rodas sobre os apoios recebidos para a prática do esporte adaptado. Caderno Brasileiro de. Terapia Ocupacional, v. 27, n. 3, p. 615-627, jul/sep. 2019.

BUFFART, L. M.; WESTENDORP, T.; VAN DEN BERG-EMONS, R. J.; STAM, H. J.; ROEBROECK, M. E. Perceived barriers to and facilitators of physical activity in young adults with childhood-onset physical disabilities. Journal of Rehabilitation Medicine, v. 41, n. 11, p. 881-885, nov. 2009.

BRAGARU, M.; WILGEN, P. C.; GEERTZEN, J. H. B.; RUIJS, S. J. B.; DIJKSTRA, P. U.; DEKKER, R. Barriers and facilitators of participation in sports: a qualitative study on Dutch individuals with lower limb amputation. PLOS ONE, v. 8, n. 3, p. 1-9, mar. 2013.

BRASIL. Ministério da Saúde. Secretaria de Vigilância em Saúde. Secretaria de Atenção à Saúde. Política Nacional de Promoção da Saúde / Ministério da Saúde, Secretaria de Vigilância em Saúde, Secretaria de Atenção à Saúde. 3. ed. Brasília: Ministério da Saúde, 2010. Disponível em: http://bvsms.saude.gov.br/bvs/publicacoes/politica_nacional_promocao_saude_3ed.pdf. Acesso em: 20 jan. 2020.

BRITTAIN, I. Perceptions of disability and their impact upon involvement in sport for people with disabilities at all levels. Journal of Sport and Social Issues, v. 28, n. 4, p. 429-452, nov. 2004.

CARDOSO, V. D. A reabilitação de pessoas com deficiência através do desporto adaptado. Revista Brasileira de Ciências do Esporte, v. 33, n. 2, p. 529-539, jun. 2011.

CENTRO DE DESPORTOS. Portal CDS, (UFSC). Florianópolis, 2018. Disponível em: https://noticias.ufsc.br/2018/11/pessoas-com-deficiencia-encontram-autonomia-e-estilo-de-vida-saudavelem-projetos-de-extensao-da-ufsc/. Acesso em: 15 fev. 2019.

ELLIS, R.; KOSMA, M.; CARDINAL, B. J.; BAUER, J. J.; MCCUBBIN, J. A. Physical activity beliefs and behaviour of adults with physical disabilities. Disability and Rehabilitation, v.29, n.15, p.12211227, aug.2007.

FAGHER, K.; LEXELL, J. Sports-related injuries in athletes with disabilities. Scandinavian Journal of Medicine \& Science in Sports, v. 24, n. 5, p. 320-331, oct. 2014.

HAYES, S. D.; CROCKER, P. R. E.; KOWALSKI, K. C. Gender differences in physical selfperceptions, global self-esteem and physical activity: evaluation of the physical self-perception profile model. Journal of Sport Behavior, v. 22, n. 1, p. 1-14. 1999. 
IBGE. INSTITUTO BRASILEIRO DE GEOGRAFIA E ESTATÍSTICA. Censo demográfico 2010. Cidades e Estados. Florianópolis, 2010. Disponível em: https://www.ibge.gov.br/cidades-eestados/sc/florianopolis.html. Acesso em: 20 ago. 2020.

JAARSMA, E. A.; DIJKSTRA, P. U.; GEERTZEN, J. H. B.; DEKKER, R. Barriers to and facilitators of sports participation for people with physical disabilities: a systematic review. Scandinavian Journal of Medicine \& Science in Sports, v. 24, n. 6, p. 871-881, dec. 2014.

JUNG, L. G.; KALINOSKI, A. X.; MARQUES, A. C. Barreiras e facilitadores para a atividade física em pessoas com déficit intelectual. Revista Brasileira de Atividade Física e Saúde, v. 22, n. 4, p. 362-372, jul. 2017.

LAMÔNICA, D. A. C.; ARAÚJO-FILHO, P.; SIMONELLI, S. B. J.; CAETANO, V. L. S. B.; REGINA, M. R. R.; REGIANI, G. M. Acessibilidade em ambiente universitário: identificação de barreiras arquitetônicas no campus da USP de Bauru. Revista Brasileira de Educação Especial, v. 14, n. 2, p. 177-188, mai/ago. 2008.

LAPE, E. C.; KATZ, J. N.; LOSINA, E.; KERMAN, H. M.; GEDMAN, M. A.; BLAUWET, C. A. Participant-reported benefits of involvement in an adaptive sports program: a qualitative study. PM \& R: The Journal of Injury, Function and Rehabilitation, v.10, n. 5, p. 507-515, aug. 2017.

LJUNGQVIST, A.; et al. The International Olympic Committee (IOC) Consensus Statement on periodic health evaluation of elite athletes. Clinical Journal of Sport Medicine, v. 19, n. 5, p. 347-365, sep. 2009.

MACKENZI, G.; WILLIS, C.; SHIELDS, N. Barriers and facilitators of physical activity participation for young people and adults with childhood-onset physical disability: a mixed methods systematic review. Developmental Medicine \& Child Neurology, feb. 2021.

MARMELEIRA, J. F. F.; FERNANDES, J. M. G. A.; RIBEIRO, N. C.; TEIXEIRA, A. T.; GUTIERRES FILHO, P. J. B. Barriers to physical activity among people with visual impairment. Revista Brasileira de Ciências do Esporte, v. 40, n. 2, p. 197-204, abr/jun. 2018.

MARTIN, J. J. Benefits and barriers to physical activity for individuals with disabilities: a socialrelational model of disability perspective. Disability and Rehabilitation, v. 35, n. 24, p. 2030-2037, jun. 2013.

MONFORTE, J.; ÚBEDA-COLOMER, J.; PANS, M.; PÉREZ-SAMANIEGO, V.; DEVÍS-DEVÍS, J. Environmental barriers and facilitators to physical activity among university students with physical disability - a qualitative study in Spain. International Journal of Environmental Research and Public Health, v. 18, n. 2, p. 464, jan. 2021.

MCLOUGHLIN, G.; FECSKE, C. W.; CASTANEDA, Y.; GWIN, C.; GRABER, K. Sport participation for elite athletes with physical disabilities: motivations, barriers, and facilitators. Adapted Physical Activity Quarterly: APAQ, v. 34, n. 4, p. 421-411, oct. 2017.

PALMA, L. E.; PATIAS, B. C.; FECK, R. M. Atividade física e deficiência física: preferências, motivações e barreiras para a prática de atividade física. Caderno de Educação Física e Esporte, v. 18, n. 2, p. 145-121, jul. 2020. 
RIMMER, J. H.; RILEY, B.; WANG, E.; RAUWORTH, A.; JURKOWSKI, J. Physical activity participation among persons with disabilities: barriers and facilitators. American Journal of Preventive Medicine, v. 26, n. 5, p. 419-425, jun. 2004.

ROLFE, D. E.; YOSHIDA, K.; RENWICK, R.; BAYLEI, C. Negotiating participation: how women living with disabilities address barriers to exercise. Health Care Women International, v.30, n. 8, p. 746-766, aug. 2009.

SERON, B. B.; ARRUDA, G. A.; GREGUOL, M. Facilitadores e barreiras percebidas para a prática de atividade física por pessoas com deficiência motora. Revista Brasileira de Ciências do Esporte, v. 37, n. 3, p. 214-221, jun. 2015.

SILVA, A. A.; et al. Esporte adaptado: abordagem sobre os fatores que influenciam a prática do esporte coletivo em cadeira de rodas. Revista Brasileira de Educação Física e Esporte, v.27, n.4, p.679-687, out/dez. 2013.

SCELZA, W. M.; KALPAKJIAN, C. Z.; ZEMPER, E. D.; Tate, D. G. Perceived barriers to exercise in people with spinal cord injury. Physical Medicine Rehabilitation, v. 84, n. 8, p. 576-583, aug. 2005.

SHIELDS, N.; SYNNOT, A. J.; BARR, M. Perceived barriers and facilitators to physical activity for children with disability: a systematic review. British Journal of Sports Medicine, v. 46, n. 14. p. 989 997, sep. 2012.

VAN DER PLOEG, H. P.; BEEK, A. J.; WOUDE, L. H. V.; MECHELEN, W. Physical activity for people with a disability: a conceptual model. Sports Medicine, v. 34, n. 10, p. 639-649, 2004.

VAN DER PLOEG, H.P. et al. Successfully improving physical activity behavior after rehabilitation. American Journal of Health Promotion, v. 21, n. 3. p. 153-159, jan./feb. 2007.

WILLIAMS, T. L.; SMITH, B.; PAPATHOMAS, A. The barriers, benefits and facilitators of leisure time physical activity among people with spinal cord injury: A meta-synthesis of qualitative findings. Health Psychology Review, v. 8, n. 4, p. 404-425, mar. 2014.

World Health Organization (WHO). Disability. 2018. Disponível em: https://www.who.int/healthtopics/disability\#tab=tab_1. Acesso em: 15 jul. 2020.

\section{Como citar este artigo}

BIDUSKI, G. M. et, al. Barreiras e facilitadores percebidos por pessoas com deficiência praticantes de esporte. Revista Kinesis, Santa Maria, v. 39, p. 01-13, 2021.

*O presente trabalho foi realizado com apoio da Coordenação de Aperfeiçoamento de Pessoal de Nível Superior - Brasil (CAPES) - Código de Financiamento 001. 